\title{
Editorial
}

\section{Fractional and Time-Scales Differential Equations}

\author{
Dumitru Baleanu, ${ }^{1}$ Ali H. Bhrawy, ${ }^{2}$ Delfim F. M. Torres, ${ }^{3}$ and Soheil Salahshour ${ }^{4}$ \\ ${ }^{1}$ Department of Mathematics and Computer Sciences, Cankaya University, TR-06810 Yenimahalle, Ankara, Turkey \\ ${ }^{2}$ Department of Mathematics, Faculty of Science, King Abdulaziz University, Jeddah 21589, Saudi Arabia \\ ${ }^{3}$ Department of Mathematics, University of Aveiro, 3810-193 Aveiro, Portugal \\ ${ }^{4}$ Young Researchers and Elite Club, Mobarakeh Branch, Islamic Azad University, Mobarakeh, Iran \\ Correspondence should be addressed to Dumitru Baleanu; dumitru@cankaya.edu.tr
}

Received 30 December 2013; Accepted 30 December 2013; Published 9 February 2014

Copyright (C) 2014 Dumitru Baleanu et al. This is an open access article distributed under the Creative Commons Attribution License, which permits unrestricted use, distribution, and reproduction in any medium, provided the original work is properly cited.

The theory and applications of fractional differential equations (FDEs) are gaining more relevance since they are used extensively in the modeling of various processes in physics, chemistry, engineering, and other areas of science. As it is known, the behavior of dynamics of most complex systems of the real world phenomena has memory. Therefore, the modeling of dynamics of these types of systems by FDEs has more advantages than the classical ones, in which such effects are neglected. On the other hand, the time-scales formalism unifies the theories of difference and differential equations. Accordingly, the time-scales analysis constitutes a good tool to study both discrete and continuous systems.

Advanced analytical and numerical techniques and computational methods are of important interest for classical, fractional, fuzzy fractional, and time-scales differential equations.

The papers of this special issue contain some new algorithms and techniques designed to investigate classical, fractional, fractal, fuzzy fractional, and time-scales differential equations of general interest. New insights of existence and uniqueness theorems of some differential equations were also presented.

In the following we summarize briefly the content of the special issue. The second Noether theorem, the local observability of systems, and the fractional Cauchy problem within Riemann-Liouville fractional delta derivative on time scales were reported and the solutions of fractional discrete systems with sequential h-differences were obtained. Besides, the oscillation criteria for fourth-order nonlinear dynamic equations on time scales were depicted. Local fractional series expansion method for wave and diffusion equations and some mappings for special functions on Cantor sets were presented. Application of fuzzy fractional kinetic equations to modeling of the acid hydrolysis reaction, the solutions of linear fractional differential equations with uncertainty, and an operational matrix based on Legendre polynomials for solving fuzzy fractional-order differential equations were also investigated. A class of fractional-order differential models of biological systems with memory to model the interaction of immune system with tumor cells and with HIV infection of $\mathrm{CD}^{+}{ }^{+}$T-cells was reported. Fractionalorder total variation image restoration based on primal-dual algorithm and the fractional dynamics of genetic algorithms using hexagonal space tessellation were pointed out. The positive solution using bifurcation techniques for boundary value problems of fractional differential equations was a contribution of our special issue. The existence results for a class of fractional differential equations with boundary value conditions and with delay, a modified generalized Laguerre spectral methods for fractional differential equations on the half line, a Jacobi collocation method for solving nonlinear Burgers-type equations, and new wavelets collocation method for solving second-order multipoint boundary value problems using Chebyshev polynomials of third and fourth kinds were shown. The investigation of the nonlinear fractional Jaulent-Miodek and Whitham-Broer-Kaup equations within Sumudu transform, the Bernstein operational matrices applied for solving the fractional quadratic Riccati differential equations, and the approximate solutions of Fisher's type equations with variable coefficients are topics covered by 
our special issue. Another part of contributions was focussing on two efficient generalized Laguerre spectral algorithms for some fractional initial value problems, the approximation of eigenvalues of Sturm-Liouville problems by using Hermite interpolation and the presentation of the numerical solutions of fractional Fokker-Planck equations by using the iterative Laplace transform method.

Dumitru Baleanu Ali H. Bhrawy

Delfim F. M. Torres Soheil Salahshour 


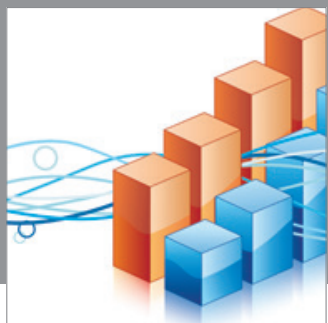

Advances in

Operations Research

mansans

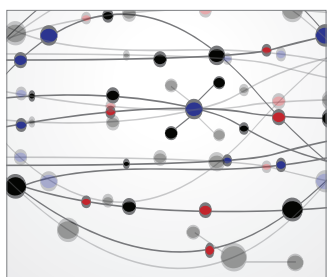

The Scientific World Journal
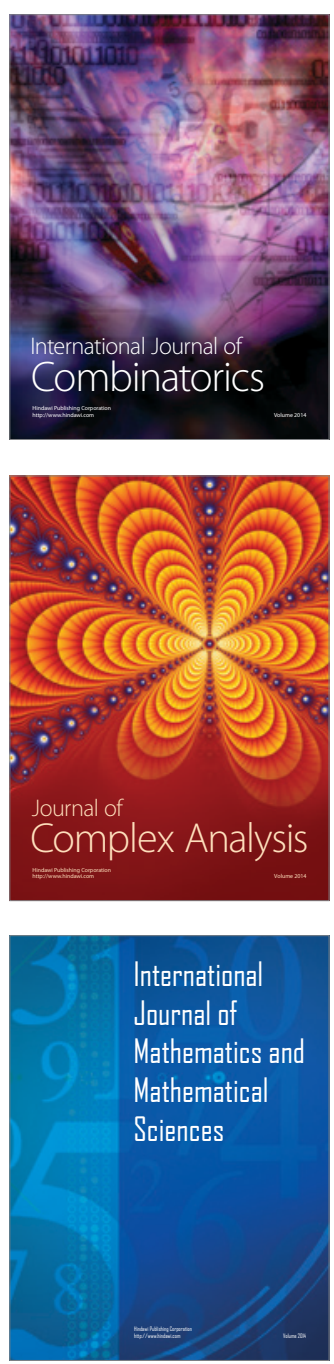
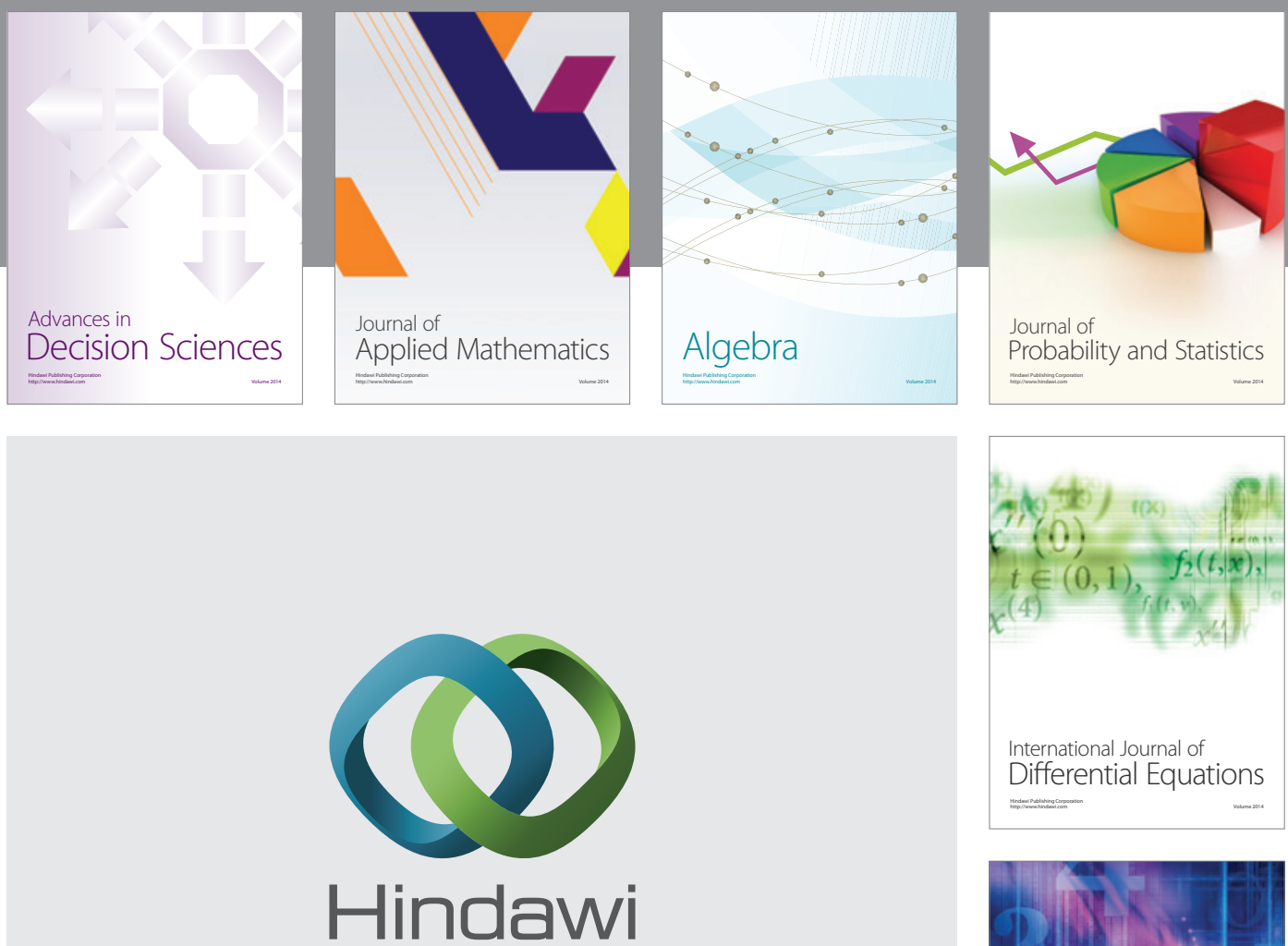

Submit your manuscripts at http://www.hindawi.com
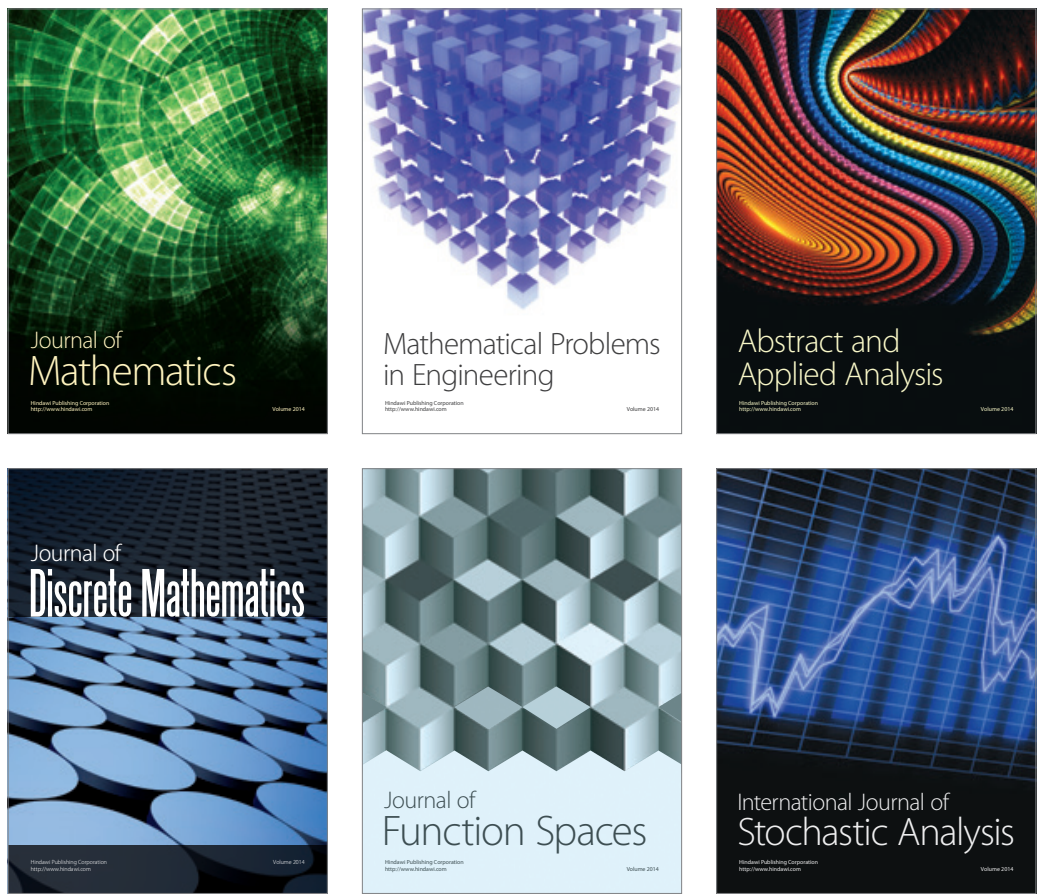

Journal of

Function Spaces

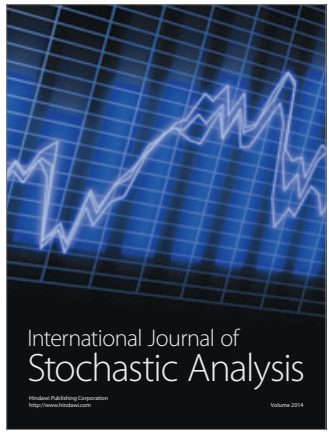

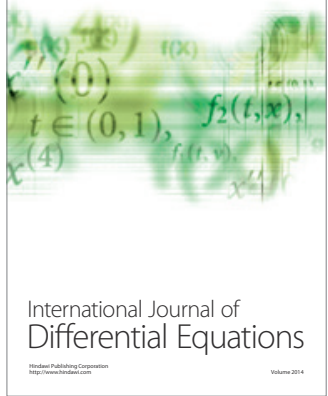
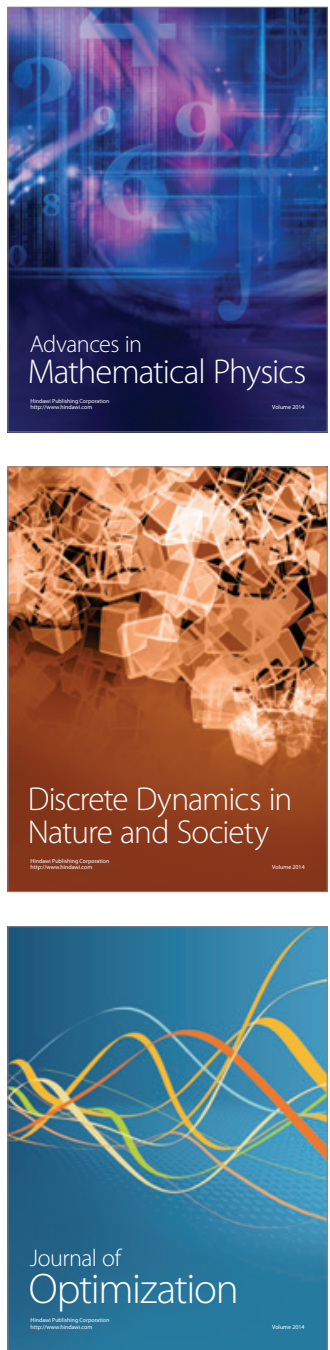